\title{
REVISING THE AGE FOR THE BAPTISTINA ASTEROID FAMILY USING WISE/NEOWISE DATA
}

\author{
Joseph R. Masiero ${ }^{1}$, A. K. Mainzer ${ }^{1}$, T. Grav ${ }^{2}$, J. M. BaueR ${ }^{1,3}$, AND R. Jedicke ${ }^{4}$ \\ ${ }^{1}$ Jet Propulsion Laboratory/California Institute of Technology, 4800 Oak Grove Dr., MS 321-520, Pasadena, CA 91109, USA; Joseph.Masiero@jpl.nasa.gov \\ ${ }^{2}$ Planetary Science Institute, 1700 East Fort Lowell, Suite 106, Tucson, AZ 85719-2395, USA \\ ${ }^{3}$ Infrared Processing and Analysis Center, California Institute of Technology, Pasadena, CA 91125, USA \\ ${ }^{4}$ Institute for Astronomy, University of Hawaii, Honolulu, HI 96822, USA \\ Received 2012 March 21; accepted 2012 September 6; published 2012 October 11
}

\begin{abstract}
We have used numerical routines to model the evolution of a simulated Baptistina family to constrain its age in light of new measurements of the diameters and albedos of family members from the Wide-field Infrared Survey Explorer. We also investigate the effect of varying the assumed physical and orbital parameters on the best-fitting age. We find that the physically allowed range of assumed values for the density and thermal conductivity induces a large uncertainty in the rate of evolution. When realistic uncertainties in the family members' physical parameters are taken into account, we find that the best-fitting age can fall anywhere in the range of 140-320 Myr. Without more information on the physical properties of the family members, it is difficult to place a more firm constraint on Baptistina's age.
\end{abstract}

Key word: minor planets, asteroids: general

Online-only material: color figures

\section{INTRODUCTION}

The Main Belt asteroids (MBAs) offer a laboratory to study the dynamical and collisional evolution of the inner solar system, as well as a window into the composition and thermal history of the protosolar disk. For nearly a century, asteroids grouped closely in orbital element space have been recognized as having formed from the catastrophic disruption of a single larger parent body (Hirayama 1918; Zappalà et al. 1990). Through modeling of the dynamical and the non-gravitational forces that evolve the orbits of the family members, the time since the breakup of the parent body has been estimated. The forces and processes that act on these small MBAs depend on the bodies' physical parameters, such as diameter and albedo. Previous modeling methods have used the absolute visible magnitudes of the family members as a proxy for their diameters (e.g., Nesvorný et al. 2005); however, this instills uncertainty in the age determination as the derived age will depend strongly on the assumed albedos. Assumptions about other thermophysical parameters will likewise introduce accompanying errors on the age determination.

The chronology of asteroid family breakups is one of the few methods, along with cratering records and petrology/radioisotope ages, for dating the history of events in the solar system. These collisional events in the Main Belt can be linked to the geological record of the Earth, as well as impacts on the terrestrial planets, other asteroids, and Earth's Moon (e.g., dell'Oro et al. 2002; O'Brien \& Greenberg 2005; Farley et al. 2006; Ćuk et al. 2010; Le Feuvre \& Wieczorek 2011). Ultimately, the goal of such analyses is to understand the sequence of events in the Main Belt and near-Earth object (NEO) populations that are known to have had major consequences for life on Earth (e.g., Alvarez et al. 1980). Finally, probing the ages of the oldest families gives us a window into the most ancient history of the solar system, as some family formation events may coincide or even predate the Late Heavy Bombardment and the epoch of giant planet migration in the solar system (Levison et al. 2001; Tsiganis et al. 2005; Morbidelli et al. 2010a, 2010b).
Until recently, diameter measurements were only available for a few thousand asteroids, most of these coming from the Infrared Astronomical Satellite survey (Tedesco et al. 2002). With the completion of the next-generation all-sky thermal infrared survey by the Wide-field Infrared Survey Explorer (WISE; Wright et al. 2010) and the identification of the small bodies of the solar system observed during that survey (the NEOWISE project; Mainzer et al. 2011a), a new data set has been opened. NEOWISE allows us to determine accurate diameters for the $>158,000$ observed Main Belt asteroids detected during the fully cryogenic portion of the WISE mission and albedos for the $>120,000$ that had previous optical measurements, of which more than 33,000 are members of previously identified asteroid families (Masiero et al. 2011). We can use these measured diameters of family members to better constrain the ages of asteroid families by revising predictions of their orbital evolution, using the methods described in Vokrouhlický et al. (2006).

However, an important consideration in any attempt to determine asteroid family age is the error introduced in that determination by the assumed values of physical and orbital parameters. Many physical parameters (e.g., macroscopic density) are only poorly constrained for more than a handful of objects, yet they play a large role in the evolution of said bodies. Similarly, the orbital parameters of the parent body at the moment of breakup can only be assumed for families older than a few million years (cf. Nesvorný \& Bottke 2004).

In this work, we address both the uncertainty due to the assumed initial conditions and the effect of using the newly available diameter and albedo data from NEOWISE to the age determination of the Baptistina asteroid family, using the work of Bottke et al. (2007) as a starting point and road map. In Section 2, we discuss the numerical routines used to model the orbital evolution, as well as the equations governing the thermal forces also acting on the body. In order to test the effect of the initial conditions chosen, we use the assumed orbital and physical parameters from Bottke et al. (2007) and vary each independently through a range of realistic values looking for changes in the fitted age from their best-fit value. We discuss 
the behavior of the fit with respect to each of these parameters in Section 3. With these effects quantified, we can then model the evolution of the family using the NEOWISE diameters and albedos. We discuss the new age determination in Section 4, and its implication in Section 5.

\section{SIMULATING ORBITAL EVOLUTION}

Under the assumption of a common location and time of origin for the members of a family, we can simulate the evolutionary history of the orbits of family members using a numerical integrator. For the Main Belt, the dominant force shaping this evolution is the gravity from the major bodies of the solar system, in particular the Sun and Jupiter. However, non-gravitational effects such as those arising from thermal radiation by the body can play an important role, particularly for the smallest MBAs. We discuss these two evolutionary forces below in the context of the software used to model them.

\subsection{SWIFT}

The dynamic evolution of minor planets due to gravitational interaction with the Sun is simulated using the Regularized Mixed Variable Symplectic integrator as implemented in the SWIFT code package (Levison \& Duncan 1994). This symplectic integrator calculates the motion of a test particle by separating its Hamiltonian into two parts: the Keplerian motion and the motion due to gravitational interaction with other bodies, each of which can be solved analytically. One Hamiltonian is applied for half a time step, the other is applied for the full time step, and the first is then applied for the remaining half-step. The Hamiltonian governing the interaction acts as an acceleration in the particles' velocity, a feature that is expanded on below when non-gravitational forces are included. This method of integration ensures that the energy of the system is conserved.

SWIFT also includes the ability to handle close-approach cases between particles at a much higher time resolution than is used for the integration in general. However, we have neglected this component of the routine to reduce total run time. As cases of close-approaches/impacts with massive bodies will remove objects from families instead of evolving them within the nominal orbital element space, this assumption will not result in a significant increase in the uncertainty of the family age. We note that (as discussed below) we do include the effect of nondestructive collisions on the reorientation of the spin states and periods of the test bodies.

Required inputs for SWIFT are the initial positions of the test particles (assumed to be all the same and coincident with the current location of the parent fragment), the initial diameters $(D)$, and the initial velocities relative to the parent. Each of the three velocity components were assigned randomly up to a maximum value that is one of the tested parameters $\left(V_{0}\right)$ and scaled inversely proportionally to the diameter of the body. For this work, we used a characteristic diameter of $5 \mathrm{~km}$, following Vokrouhlický et al. (2006), to allow for comparison with previous results. We compare our simulations with the observed family using two different methods of diameter determination (depending on the goal of the simulation, as discussed below). For simulations that were compared to family lists generated from the optically selected population (and thus without diameter information), we used a single assumed albedo for the entire family and estimate diameters from the $H$ absolute magnitude and the albedo. For comparisons to the families identified in the WISE data (Masiero et al. 2011), we use the diameters and albedos drawn from that work. Diameters from WISE were measured independently of other sources of data, however the albedo measurements required a literature $H$ magnitude and so are subject to optical observation biases and errors. It is important to note that the family lists used in Masiero et al. (2011) were drawn from Nesvorný et al. (2006) who determined family membership from a sample of optically discovered asteroids; it is expected that small, low albedo asteroids will be underrepresented in these family lists, and that this may alter the determination of family age. Including asteroids discovered by WISE will begin to mitigate this problem, and this will be the subject of future work.

\subsection{SWIFT_RMVSY}

To account for the non-gravitational forces due to thermal emission, we use the SWIFT_RMVSY modification of the SWIFT code (Brož 2006). This upgrade uses the equations derived by Vokrouhlický $(1998,1999)$ and Vokrouhlický \& Farinella (1999) to describe the thermal forces acting on small solar system objects. When the thermal force modifies the orbit of a body it is known as the Yarkovsky effect, and it occurs when incident optical light is absorbed by a surface and re-emitted as thermal infrared radiation in a different direction due to the rotation of the body (see Bottke et al. 2006, for a complete discussion). The Yarkovsky-O'Keefe-Radzievskii-Paddack (YORP) effect models the way thermal radiation can change the spin state of non-spherical bodies without atmospheres (Rubincam 2000).

To calculate these thermal forces, SWIFT_RMVSY requires an input of the thermal and physical parameters for each object: diameter, visible geometric albedo $\left(p_{V}\right)$, thermal conductivity $(K)$, thermal capacity $\left(C_{p}\right)$, infrared emissivity $(\epsilon)$, surface density $\left(\rho_{s}\right)$, bulk density $(\rho)$, rotation rate $(\omega)$, and rotation pole orientation. As a starting point for comparisons with the WISE data, we assumed values of $K=0.01 \mathrm{~W} \mathrm{~m}^{-1} \mathrm{~K}^{-1}$, $C_{p}=680 \mathrm{~J} \mathrm{~kg}^{-1} \mathrm{~K}^{-1}, \epsilon=1$, and $\rho=\rho_{s}=2200 \mathrm{~kg} \mathrm{~m}^{-3}$, and assigned the population random rotation rates and poles, following Vokrouhlický et al. (2006). For comparisons with literature work, we use the same values assumed there. We discuss below the effects of varying these parameters on the best-fitting age.

As an object in the Main Belt evolves over time, it is predicted that it will undergo small, non-disruptive impacts that can change the body's rotation state (both spin pole and rotation period), occurring with a characteristic timescale depending on diameter and rotational angular momentum. We have modified the SWIFT_RMVSY code to account for this collisional reorientation by using the characteristic time of reorientation $\left(\tau_{r}\right)$ described by Vokrouhlický et al. (2006):

$$
\tau_{r}=B\left(\omega / \omega_{0}\right)^{\beta_{1}}\left(D / D_{0}\right)^{\beta_{2}},
$$

where $B=84.5 \mathrm{kyr}, \beta_{1}=5 / 6, \beta_{2}=4 / 3, D_{0}=2 \mathrm{~m}$ (i.e., a radius of $1 \mathrm{~m}$; see Farinella et al. 1998), and $\omega_{0}$ corresponds to a period of five hour (near the peak in the debiased distribution of MBA rotation rates; see Masiero et al. 2009). In addition to reorienting spin poles, we also allow collisions to reset the rotation rate of the body in a random fashion.

While collisional reorientation is treated as a random event, the gradual reorientation of the spin axis by the YORP effect is treated as a continuous change, preferentially driving the rotation pole toward an asymptotic limit of $0^{\circ}$ or $180^{\circ}$ (Vokrouhlický \& C̆apek 2002). We use the median reorientation rate $\left(d \epsilon / d t=8.6 \mathrm{deg} \mathrm{Myr}^{-1}\right)$ and period doubling/halving time 

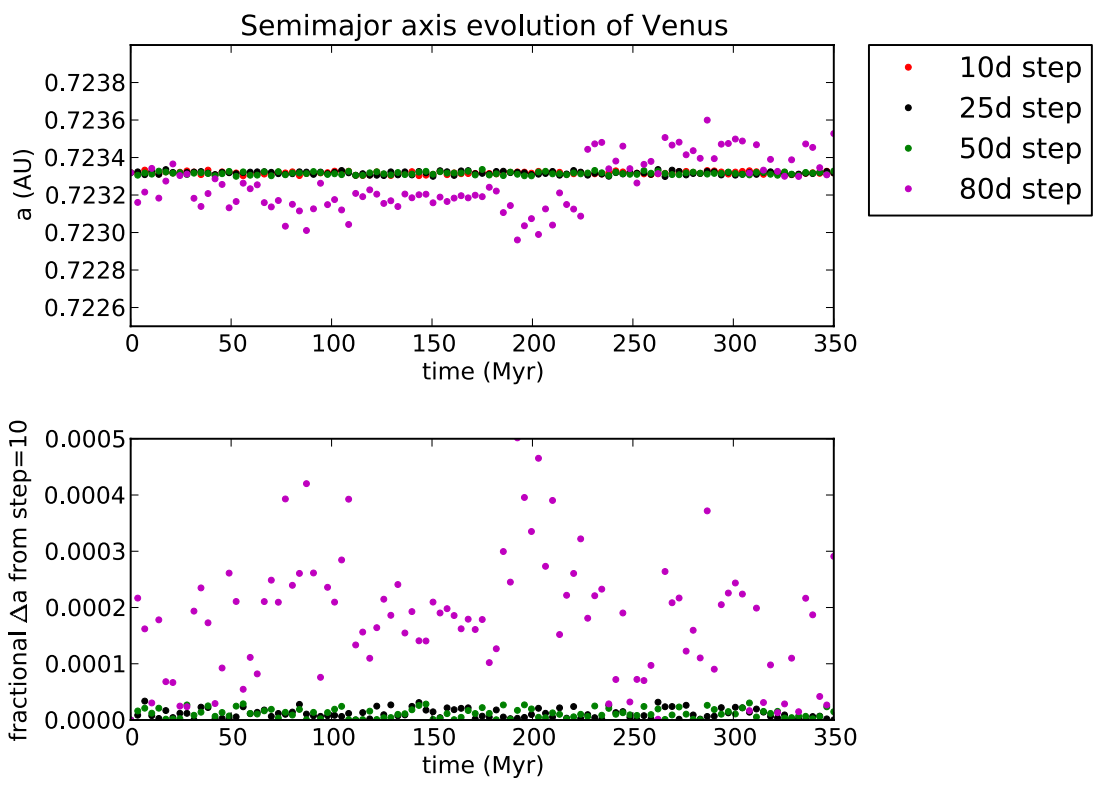

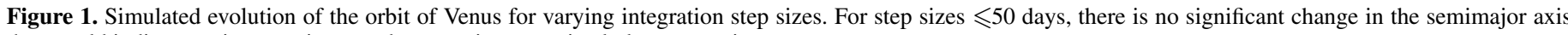
that would indicate an increase in error due to an inappropriately large step size.

(A color version of this figure is available in the online journal.)

$\left(\tau_{\text {per }}=11.9 \mathrm{Myr}\right)$ derived from thermophysical simulations of test bodies by C̆apek \& Vokrouhlický (2004) for the thermal conductivity matching our assumed value above $(K=0.01)$. We note that we scale these timescales by the rotation rate as discussed by those authors. Following Vokrouhlický et al. (2006), we also include a multiplicative parameter $c_{\text {YORP }}$ that is applied to both YORP parameters above $\left(\tau_{\text {per }}^{\prime}=\left(11.9 / c_{\text {YORP }}\right)\right.$ and $\left.d \epsilon / d t^{\prime}=c_{\text {YORP }} \times 8.6\right)$ to model the uncertainty in the age due to the weakly constrained YORP model. This parameter has been previously found to only show a weak effect on the age determination (cf. Vokrouhlický et al. 2006; Bottke et al. 2007) as long as it is non-zero, though we discuss our findings further below.

\subsection{Supercomputing Resources}

Our numerical simulations make use of the supercomputing resources available at NASA's Jet Propulsion Laboratory. We used the Zodiac supercomputer, comprised of 64 12-core Altix $2.66 \mathrm{GHz}$ nodes, for all simulations discussed here. Zodiac uses a 88 terabyte Lustre parallel filesystem allowing for improved I/O capability, especially for rapid writing to multiple files. Total peak performance is over 19 teraflops. The range of simulations shown here required approximately $300,000 \mathrm{CPU}$ hours of run time.

\subsection{Integration Step Size}

For all of the simulations we discuss in this manuscript, we included as massive particles Venus, Earth, Mars, Jupiter, and Saturn, in addition to the test particles and the Sun. Uranus and Neptune are omitted as they should play a much less significant role in the test particle evolution than Jupiter and Saturn. As Venus has the smallest semimajor axis and perihelion of any tested body (with the exception of MBAs ejected from the Belt into the NEO population, which are no longer considered family members and hence are ignored once ejected), our step size is restricted by Venus' orbital period. It is canonically recommended that the integration step size for a

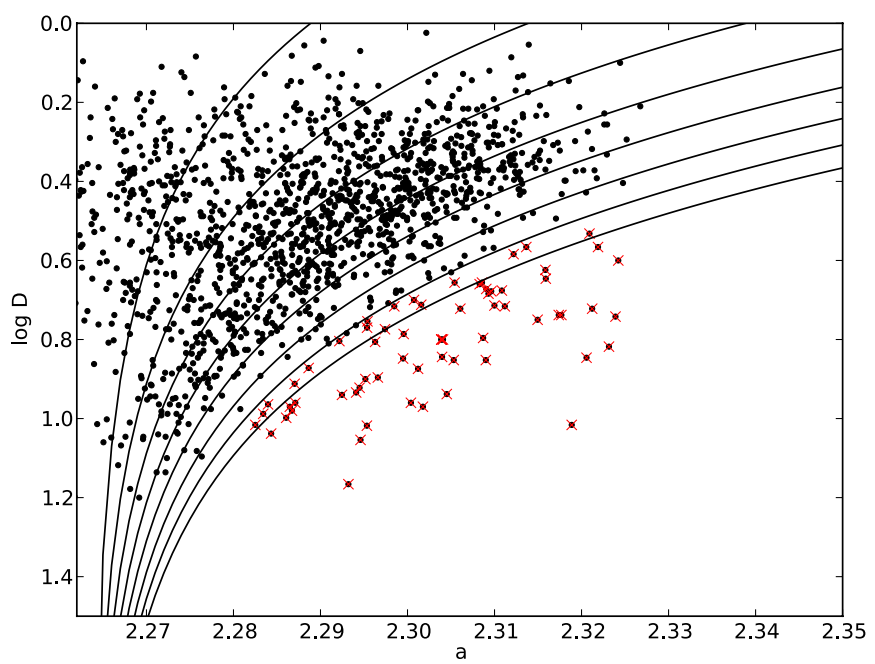

Figure 2. Diameter vs. semimajor axis for the Baptistina family members used in our analysis. The black lines show evenly spaced steps of the C-parameter (see Section 2.6) used to compare family distributions, and points overlaid with a red " $x$ " were assumed to be background objects and were not included in our analysis.

(A color version of this figure is available in the online journal.)

symplectic integrator be $\lesssim 10 \%$ of the period of the innermost body (assuming a circular orbit) to prevent a rapid accumulation of error on the total system energy (e.g., Brož 2006). We have tested the effect of step size on the simulated evolution, and show in Figure 1 the semimajor axis of Venus as a function of time for step sizes of 10, 25, 50, and 80 days, as well as the fractional change. If the step size is inappropriately large, then we should see deviations in the evolution of Venus from the shortest timestep tested. For step sizes $\leqslant 50$ days, we see no significant changes in the evolution of Venus with respect to the 10 day step simulation. For the remaining simulations in this work, we use a step size of 25 days to ensure that we are well within the range of acceptable step sizes, finding it to be the best balance between integration accuracy and time required to perform the simulations. 

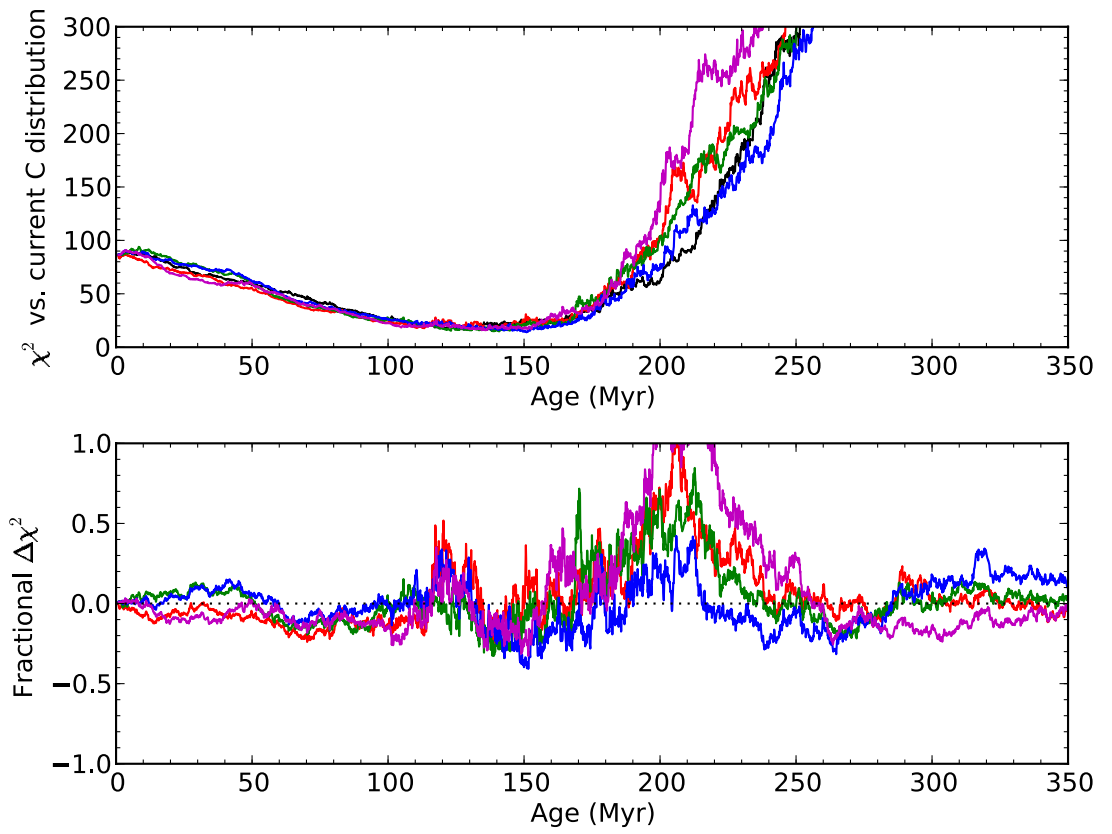

Figure 3. Identical simulations of the evolution of the Baptistina family changing the initial, random spin states of the test particles. The lower plot shows the fractional difference between the first test and the other four, for comparison.

(A color version of this figure is available in the online journal.)

\subsection{Family Membership}

In order to determine the most accurate age possible for the family, the list of family members that the simulations will be compared to must have minimal corruption from asteroids that dynamically link to the family but are not members. This is a particular problem for the Baptistina family, as the branch of the family that extends to smaller semimajor axes overlaps with the much larger and older Flora family (cf. Nesvorný et al. 2002).

Following Bottke et al. (2007), we restrict our analysis to consider only the Baptistina family members at semimajor axes larger than the parent body. We have accomplished this by using the Hierarchical Clustering Method (HCM; Zappalà et al. $1990,1994)$ of family identification to test a range of cutoff velocities. We choose the highest velocity that did not link to the lower-semimajor axis wing $\left(39 \mathrm{~m} \mathrm{~s}^{-1}\right)$ as our cutoff for family membership, following Bottke et al. (2007). Likewise, we have removed from our list linked objects that are both large and distant from the parent, and thus have a high probability of being incorrect associations. In Figure 2, we show the resultant HCM-derived family that we use in our analysis. Objects that were rejected from the list are shown overlaid with an "x." We note that while this will reduce uncertainty due to incorrectly identified family members, it also decreases the sample size of WISE-measured asteroids to 360 objects and impacts our ability to accurately compare the models to the true distribution. Identification of new family members and measurement of their physical parameters will help us decrease these uncertainties.

\subsection{Goodness of Fit Determination}

We cannot uniquely link individual test particles to observed family members as the randomized initial conditions will not necessarily mean the evolutions are identical. Instead, we focus on the distribution of the true and test populations to find the best matching initial conditions. To compare our simulation to the known population, we perform a $\chi^{2}$ test of the $C$ parameter, which is defined as $C=\Delta a 10^{-0.2 H}$ by Vokrouhlický et al. (2006) for cases where the albedo is unknown. For tests conducted using only objects with physical parameters measured by WISE, we define a $C_{D}$ parameter as $C_{D}=\Delta a D$ (where $D$ is the diameter) that is roughly equivalent to the $C$ parameter with a multiplicative offset. Larger objects are predicted to have smaller drift rates from non-gravitational effects, and so the $C$ and $C_{D}$ parameters represent lines of constant time for a given drift strength. An important difference is that $C_{D}$ has no dependence on the albedo of the asteroid, unlike $C$.

Figure 2 shows a series of curves indicating $C_{D}$ values from 0.025 to 0.2 in steps of 0.025 overlaid on the Baptistina family. To compare simulations to reality, we compare the $C$ or $C_{D}$ distribution of the simulation to the same distribution for the family. As discussed above, we only use family members at semimajor axes larger than the parent, and thus likewise only consider simulated particles that are in that same region of semimajor axis-space at the time step being tested. We note that it is possible for a particle to begin the simulation drifting inward and later through reorientation begin moving outward. Thus, it is possible for that particle to be used for the comparison to the observed family members at some time steps but not others. The goodness of fit at each time step is obtained from a binby-bin $\chi^{2}$ comparison of the two populations. The match to the observed data initially improves as the test bodies disperse over time, until they expand beyond the observed population and the $\chi^{2}$ climbs. The time at which the minimum $\chi^{2}$ is reached is therefore the best fit to the present day family, and thus can be inferred to be the age of the family.

\section{ERRORS DUE TO ASSUMED PHYSICAL AND ORBITAL PARAMETERS}

The numerical simulations of the orbital evolution of family members are deterministic in the sense that the equations of motion (both gravitational and non-gravitational) can be described analytically. However, the specific behavior of an 

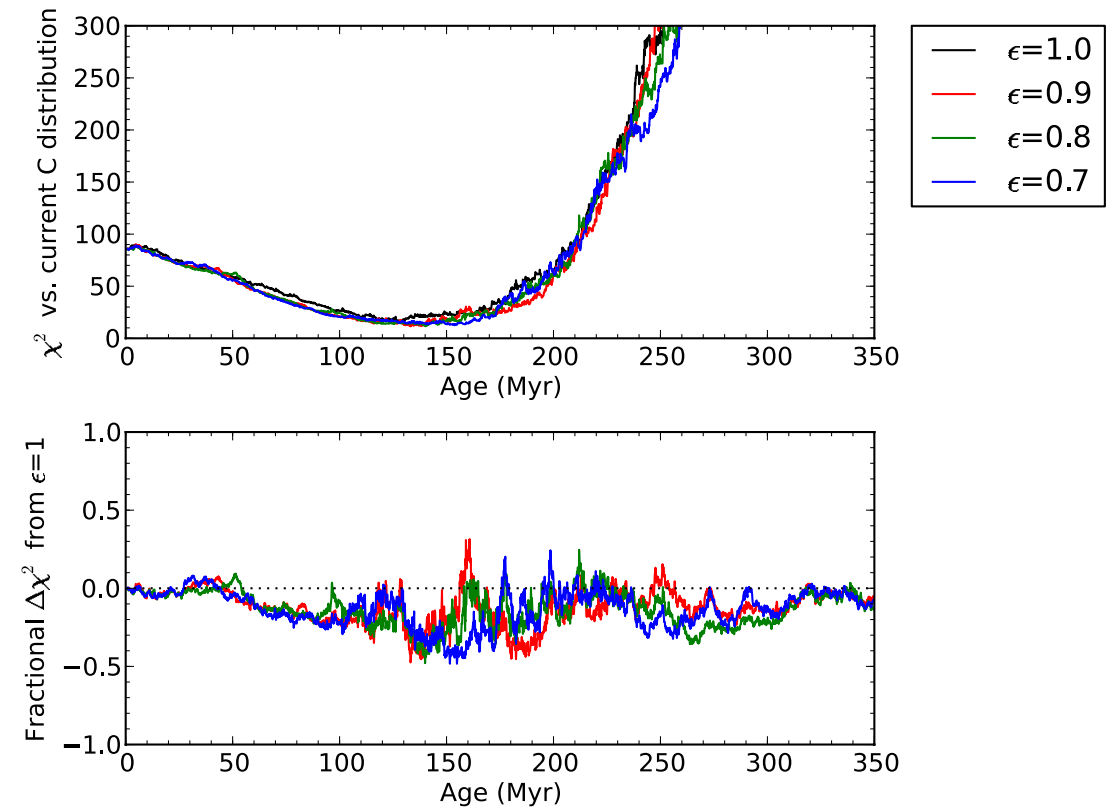

Figure 4. Same as Figure 3, but now testing various values of emissivity $(\epsilon)$. The lower plot shows the fractional difference between the $\epsilon=1$ case and the other tests, for comparison.

(A color version of this figure is available in the online journal.)

individual particle depends strongly on the initial conditions assumed for it, including the physical, orbital, and spin state parameters. While the effect of the randomized initial conditions on the behavior of the population should fade as the population of test particles grows (e.g., the initial spin pole and rotation rate, the randomized collisional reorientation of particles, etc.), other initial conditions that are singularly chosen for the population and do not change with time may have a dramatic effect on the overall evolution.

Before attempting to fit ages for asteroid families, we first will test our dependence on the chosen value for each parameter. We constrain the possible errors induced by assumptions of the thermal parameters $\left(K, \epsilon, C_{p}\right)$, orbital parameters (mean anomaly, longitude of perihelion, longitude of the ascending node), and physical parameters ( $\rho$, spin state). We include $V_{0}$ and $c_{\text {YORP }}$ as tested parameters that are varied to find the best-fitting age, and so will not discuss them here. Additionally, it is beyond the scope of the work presented here to investigate the effect of varying the equations governing the velocity distribution of the impact ejecta (here assumed to be $V=V_{0}(5 \mathrm{~km} / D)$ ) and collisional reorientation (Equation (1)), however these also will act as a source of uncertainty.

For the tests of the physical and orbital parameters, we follow the assumed initial conditions and albedo for the Baptistina family from Bottke et al. (2007) for the purpose of comparison. Once the uncertainty due to the assumed initial conditions has been quantified, we conduct a new set of simulations that use the measured values for the diameters and albedos in Section 4 to update the age of the Baptistina family.

Following the best-fit values from Bottke et al. (2007) for Baptistina, we assume a breakup velocity for the parameter tests of $V_{0}=40 \mathrm{~m} \mathrm{~s}^{-1}, c_{\text {YORP }}=1.0, K=0.01 \mathrm{~W} \mathrm{~m}^{-1} \mathrm{~K}^{-1}$, $C_{p}=680 \mathrm{~J} \mathrm{~kg}^{-1} \mathrm{~K}^{-1}, \epsilon=1, \rho=\rho_{s}=1300 \mathrm{~kg} \mathrm{~m}^{-3}$, and randomized rotation states. We note that using identical initial conditions, we reproduce the best-fitting age of $T \sim 160 \mathrm{Myr}$ for the family found by those authors. In order to compare our results directly to previous work, we initially use the $H$ magnitudes along with the assumed albedo used by those authors $\left(p_{V}=0.05\right)$. In Section 4, we use the WISE-measured diameters and albedos.

\subsection{Rotation State}

The assumed initial rotation pole and period of a test particle will dictate the magnitude and direction of the Yarkovsky force at the outset of the simulation. Over the course of the evolution of the family, the YORP effect will gradually reorient the spin axis of a test particle and slow or speed its rotation (Čapek \& Vokrouhlický 2004), while collisions will occasionally abruptly randomize these values. While YORP, by driving the rotation poles to obliquities of $0^{\circ}$ or $180^{\circ}$, will in general increase the magnitude of the Yarkovsky effect, collisions are more likely to decrease its strength or reverse it completely.

In Figure 3, we show five identical simulations of the Baptistina family, allowing only the randomized spin states of the test particles to vary. The evolution of these simulations varies in $\chi^{2}$ by $\sim 25 \%$ for the first 175 Myr. After this point, when the comparisons between the simulations and the real distribution become rapidly worse, the differences between simulations increases, however this regime is less deterministic of age of the family. This results in an uncertainty in the specific best-fit age of $\sim 20 \mathrm{Myr}$ in the case of Baptistina, however the range of likely ages remains comparable.

\subsection{Thermal Properties}

The thermal parameters of the test particles can have a significant effect on the evolution of the family. We therefore have tested the effect of altering the assumed thermophysical parameters on the evolution of the test population. In particular, we focus on varying $\epsilon, K$, and $C_{p}$ across ranges typical for realworld materials around the default assumed values of $\epsilon_{0}=1.0$, $K_{0}=0.01 \mathrm{~W} \mathrm{~m}^{-1} \mathrm{~K}^{-1}$, and $C_{p, 0}=680 \mathrm{~J} \mathrm{~kg}^{-1} \mathrm{~K}^{-1}$.

We show in Figure 4 the evolution of the Baptistina test family for various initial emissivity values, over the range of 

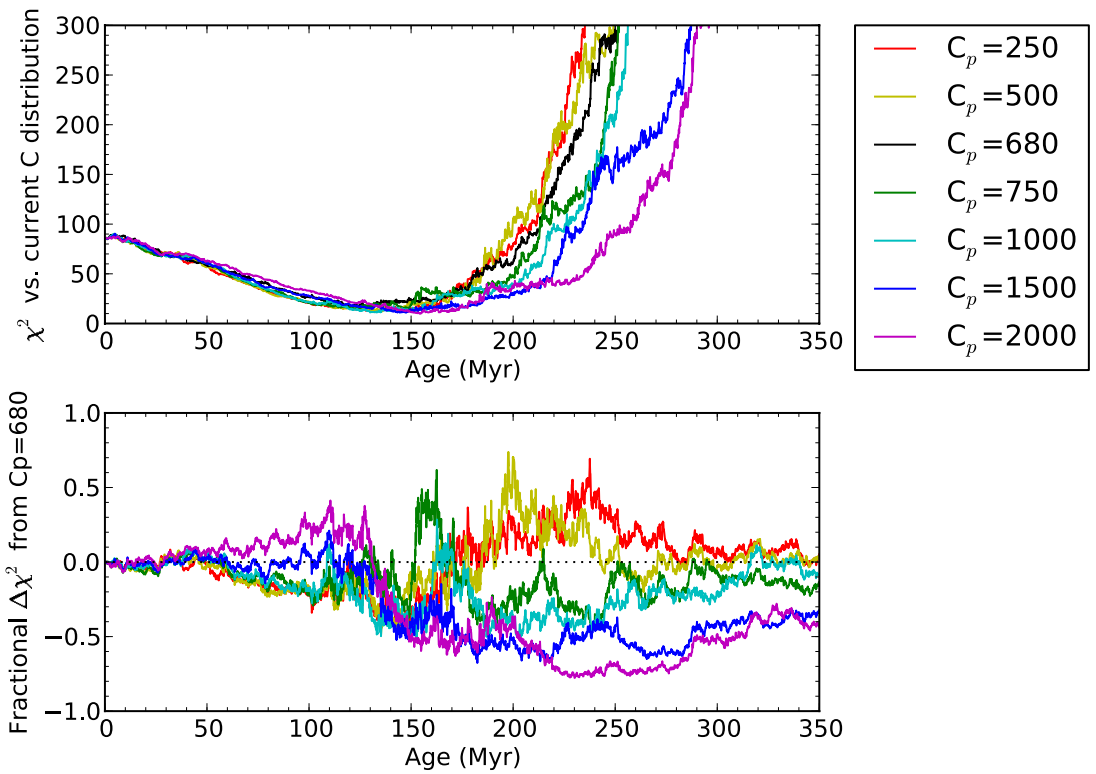

Figure 5. Same as Figure 3, but now testing various values of thermal capacity $\left(C_{p}\right)$. The lower plot shows the fractional difference between the $C_{p}=680 \mathrm{~J} \mathrm{~kg}^{-1} \mathrm{~K}^{-1}$ case and the other tests, for comparison.

(A color version of this figure is available in the online journal.)

$0.7 \leqslant \epsilon \leqslant 1.0$. We see no significant differences between each of the cases when only thermal emissivity is varied. Thus, our assumed value for emissivity of $\epsilon=1.0$ is valid for future tests. Likewise, in Figure 5, we show the evolution of the test family comparing a wide range of different thermal capacities: $250<C_{p}<2000 \mathrm{~J} \mathrm{~kg}^{-1} \mathrm{~K}^{-1}$. We again see no significant changes at ages less than 150 Myr. Beyond this age, the simulations appear to sort roughly corresponding to $C_{p}$, where simulations with smaller values of $C_{p}$ diverge from the real population faster than those with larger $C_{p}$. For the purposes of finding the best-fit age, an assumed value of $C_{p}=680 \mathrm{~J} \mathrm{~kg}^{-1} \mathrm{~K}^{-1}$ is adequate.

Conversely, we find that the assumed value of thermal conductivity $(K)$ has a significant impact on the strength of the thermal forces acting on the bodies, as it is the only parameter that varies over many orders of magnitude in realistic materials. Vokrouhlický (1998) show in their Figure 3 the relative strength of the transverse Yarkovsky force vector as a function of the thermal parameter $\Theta$; they use $K=0.01 \mathrm{~W} \mathrm{~m}^{-1} \mathrm{~K}^{-1}$ and the nominal assumptions for $C_{p}, \epsilon, \rho$, and rotation rate places $\Theta$ at the peak value for the transverse force. Changes in $K$ by half or one order of magnitude result in a significant change in the strength of the Yarkovsky effect. Following the thermal inertias $(\Gamma)$ found by (Delbo \& Tanga 2009) for asteroids with $D<200 \mathrm{~km}$, we test a range of thermal inertia values of $40<\Gamma<1200 \mathrm{~J} \mathrm{~s}^{-0.5} \mathrm{~m}^{-2} \mathrm{~K}^{-1}$ which corresponds to thermal conductivities of $0.001<K<1$ for nominal values of density and thermal capacity. We show the results of these simulations in Figure 6. The evolution of the test family is significantly slower for values both larger and smaller than $K=0.01 \mathrm{~W} \mathrm{~m}^{-1} \mathrm{~K}^{-1}$. We note that while $K \sim 1$ is only observed for the smallest of near-Earth asteroids that are believed to have surfaces free of regolith, and thus may not be a good analog for $D \sim 5 \mathrm{~km}$ MBAs, the range of $0.001<K<0.1$ is still possible for MBAs. We use $K=0.01 \mathrm{~W} \mathrm{~m}^{-1} \mathrm{~K}^{-1}$ for future simulations, however this probably represents only a lower limit on the family age. Determination of thermal conductivity or thermal inertia for a number of family members will be critical to determining the true evolution of the family.

\subsection{Initial Orbit}

In order to model the breakup of a family, we assume that all members began at the same place in space and time, and assign them an ejection velocity that scales inversely with their diameter (following Vokrouhlický et al. 2006), which combines with the particle's velocity around the sun to generate a new orbit. As the ejection velocities typically are small compared to the motion around the sun, this will preferentially elongate the cloud along the path of the orbit. Although the velocity imparted on the fragments by the collision will be randomized around a constant value, for a parent body with an eccentric orbit the change in orbital parameters after the impact can vary depending on the parent's mean anomaly at the time of breakup. Nominally, we use the present day osculating orbital elements for the largest family member as the orbit of the body prior to breakup, ensuring that the test particles are in the same osculating system as the planets (including using the same assumed epoch). However, we have tested the results of varying the mean anomaly (MA), longitude of perihelion ( $\varpi)$, and longitude of the ascending node $(\Omega)$ on the subsequent evolution of the family.

In Figure 7, we show a range of simulations with identical physical parameters, $c_{\text {YORP }}$ and breakup velocity $V_{0}$, while stepping through mean anomaly of the parent at the time of breakup. The velocity added to a test particle's motion upon breakup alters its initial orbit. However, the initial impulse is more effective at changing the orbit's aphelion when the breakup is at perihelion than it is at changing the orbit's perihelion when the breakup is at aphelion. This effect is shown in Figure 7 as the offset in $\chi^{2}$ at $T=0$, where simulations with breakups closer to perihelion have a larger initial spread in semimajor axis and thus a lower $\chi^{2}$.

In general, after about $\sim 100 \mathrm{Myr}$, the differences between populations with different initial mean anomalies are erased by the effect of Yarkovsky-induced drifts and gravitational orbital evolution. We note that this timescale will depend on the initial eccentricity of the parent body: parents with low or zero eccentricity should see little difference in family member distribution between breakups at perihelion or aphelion even 


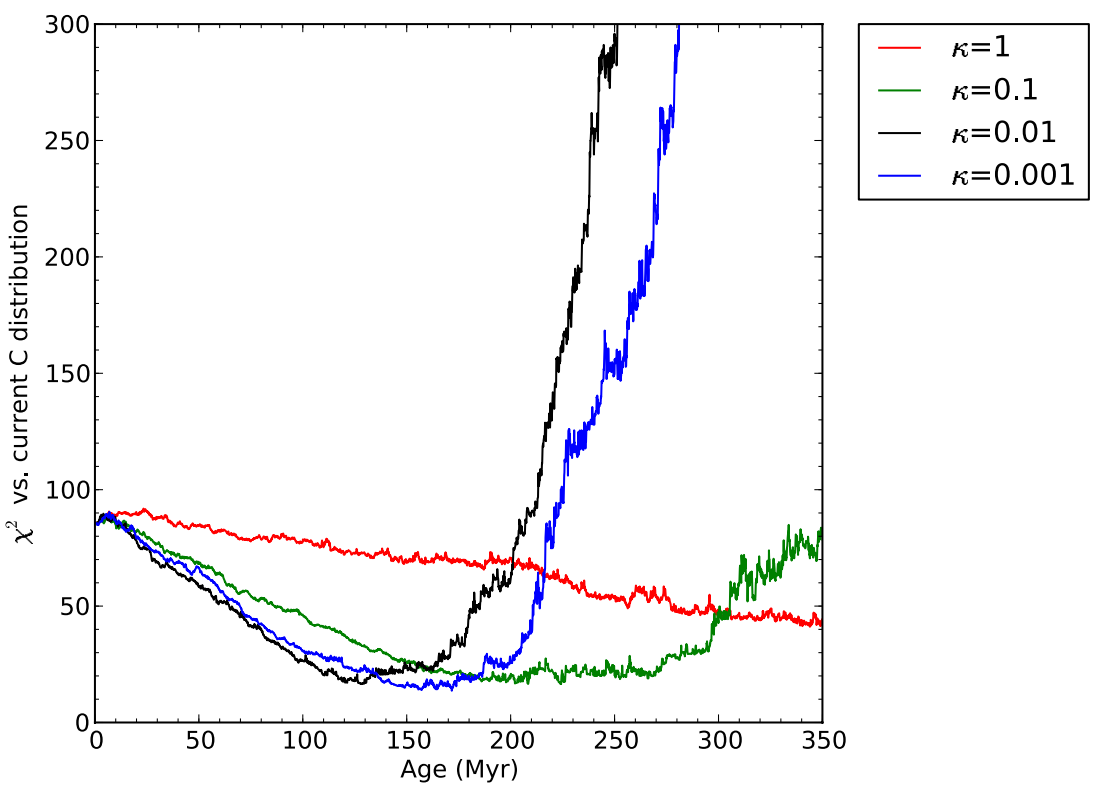

Figure 6. Same as Figure 3, but now testing various values of thermal conductivity $(K)$.

(A color version of this figure is available in the online journal.)
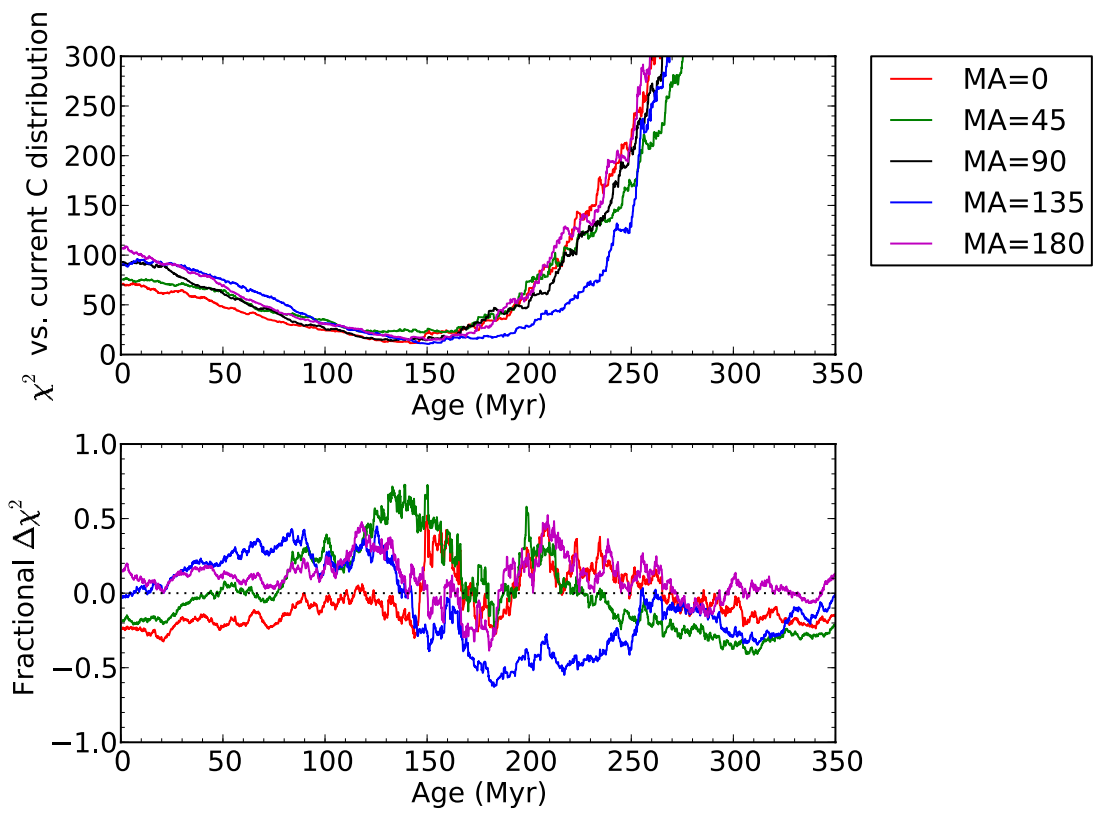

Figure 7. Same as Figure 3, but now testing a range of MA values at the time of breakup.

(A color version of this figure is available in the online journal.)

at $T=0$, while those with larger eccentricities will require more time to erase the initial differences. This effect may thus be particularly important for high-eccentricity families younger than $\sim 100$ Myr.

We show in Figures 8 and 9 the results of similar simulations, testing $\varpi$ and $\Omega$ respectively. Variations in both parameters result in no significant change to the evolution of the population in general. While these parameters may have an effect for other families with parents significantly more eccentric than Baptistina, we can safely use the present-day osculating values for all parameters for the simulations we discuss in Section 4.

\subsection{Density}

A key assumption in determining the strength of the Yarkovsky effect on the orbit of an asteroid is the mass of the body. The Yarkovsky effect is expected to produce a force that depends on the illuminated area of the body, but the resultant acceleration will scale with the mass. While the SWIFT_RMVSY code includes a parameter to allow for testing the variation in the strength of the YORP effect due to the uncertainty in its absolute strength (the $c_{\text {YORP }}$ parameter), the Yarkovsky effect should be well quantified if the mass and thermal parameters are known and thus does not include this scaling parameter. With WISE, we can usually derive effective diameters to within $\sim 10 \%$ for asteroids observed with good signal-to-noise ratio (Mainzer et al. 2011 b). However, the bulk density of asteroids remains poorly constrained. Likewise, surface density, which is a component of the calculation of thermal propagation in Yarkovsky, is equally difficult to determine and is assumed here to be equal to the bulk density. 

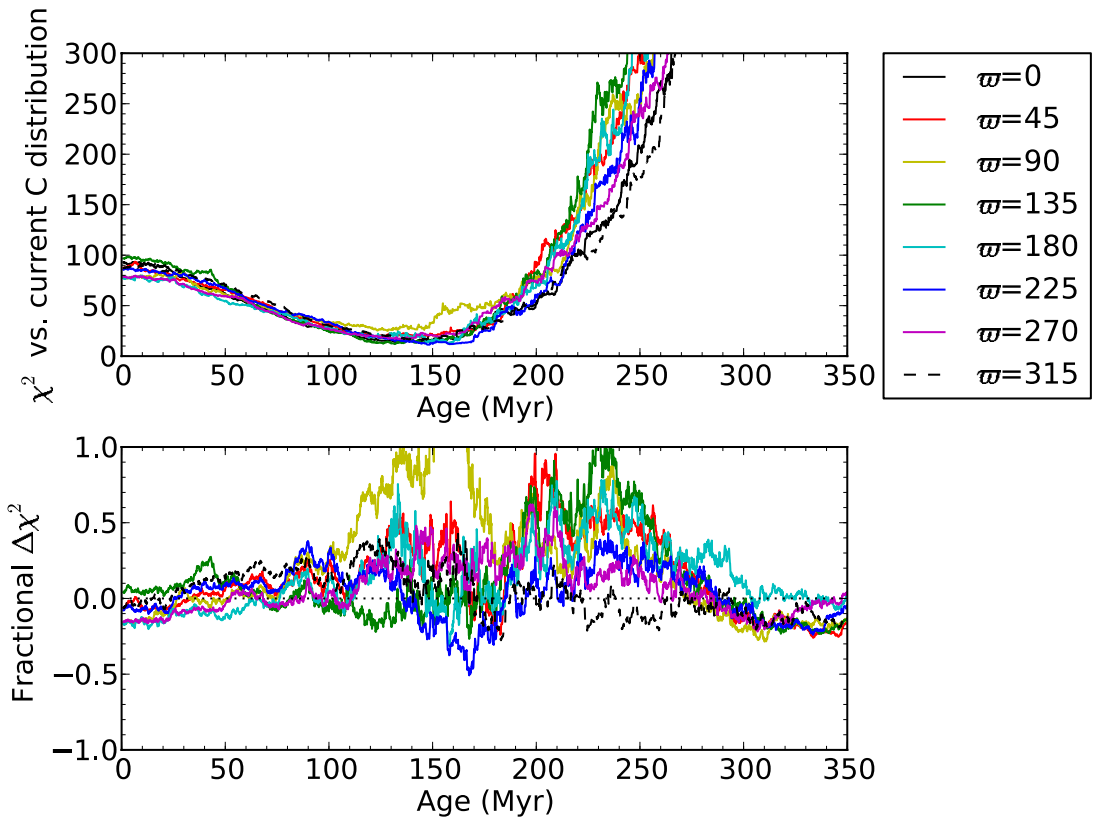

Figure 8. Same as Figure 3, but now testing a range of values for the longitude of perihelion $(\varpi)$ at the time of breakup. (A color version of this figure is available in the online journal.)
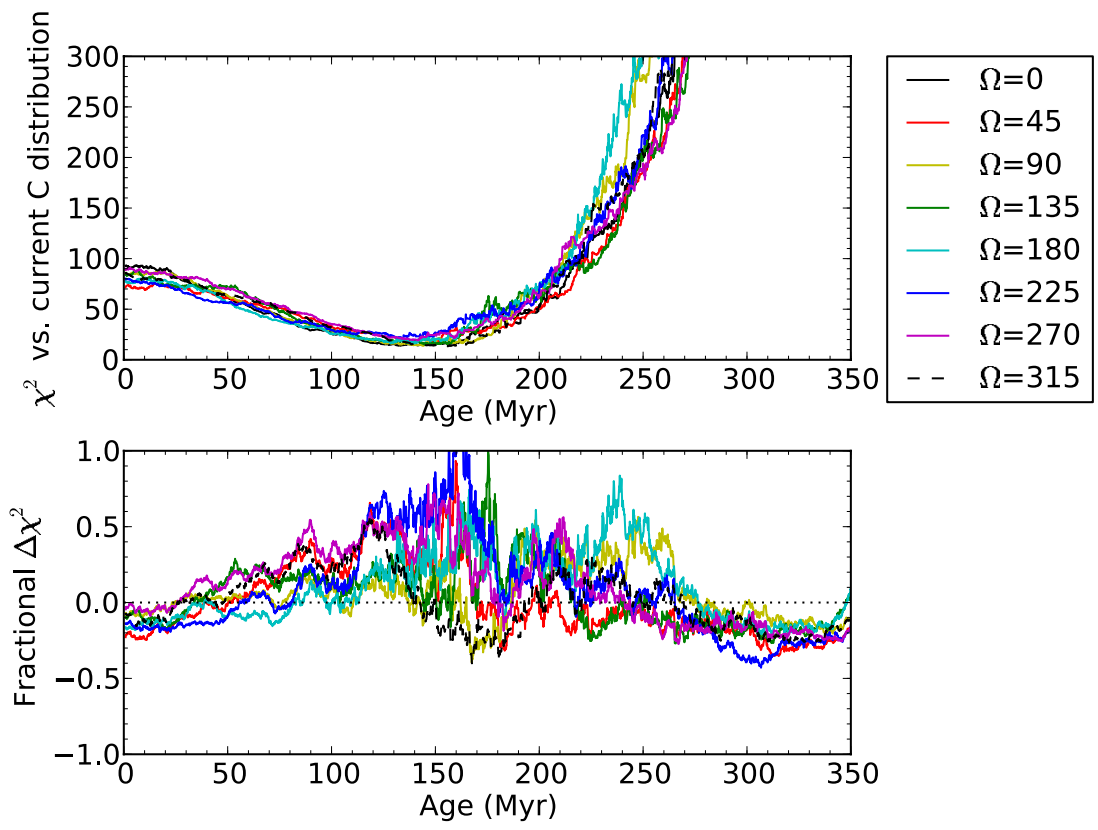

Figure 9. Same as Figure 3, but now testing a range of values for the longitude of the ascending node $(\Omega)$ at the time of breakup.

(A color version of this figure is available in the online journal.)

Density measurements of meteorites can provide an upper limit to the density we expect for different compositions of asteroid, but linking meteorites to asteroids can be difficult, and the macro- and micro-porosity of a body (which will strongly affect the measured bulk density) are almost impossible to measure remotely. Conversely, asteroid masses can be obtained from their gravitational perturbation of the other objects in the Main Belt (for the few largest bodies), from deviations on spacecraft trajectories during fly-by (for the handful of objects visited by spacecraft), or from the periods of satellite bodies in orbit around the asteroid of interest (if satellites are known to exist and the periods can be measured).
Carry (2012) provides a thorough review of the state of knowledge of asteroid densities. They list densities for 38 MBAs smaller than $D=200 \mathrm{~km}$ and with density accuracy better than $20 \%$. The mean density of this group is $\rho=2.3 \pm 1.2 \mathrm{~g} \mathrm{~cm}^{-3}$, however, the error is inflated by the range of compositions. Attempting to trace composition with spectral taxonomy, they show that the range of bulk density within a given spectral taxonomic class can still be large, due to changes in macroporosity which they attribute to increasing compaction at larger diameters. The authors show some correlation between density and spectral type (though even then the intrinsic scatter is about $\sim 25 \%$ in the best cases) and find similar discrepancies to the 


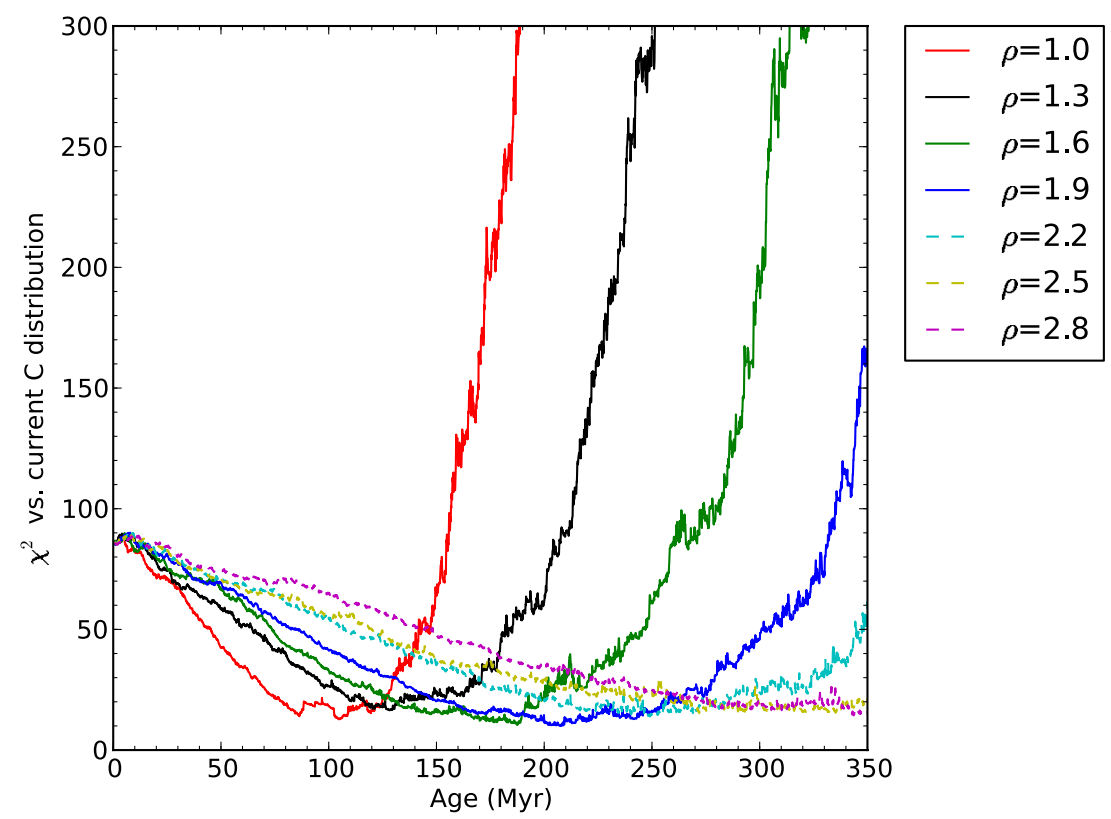

Figure 10. Simulations of the evolution of the Baptistina family under varying assumptions for the bulk and surface density of the test particles (assuming both densities are equal).

(A color version of this figure is available in the online journal.)
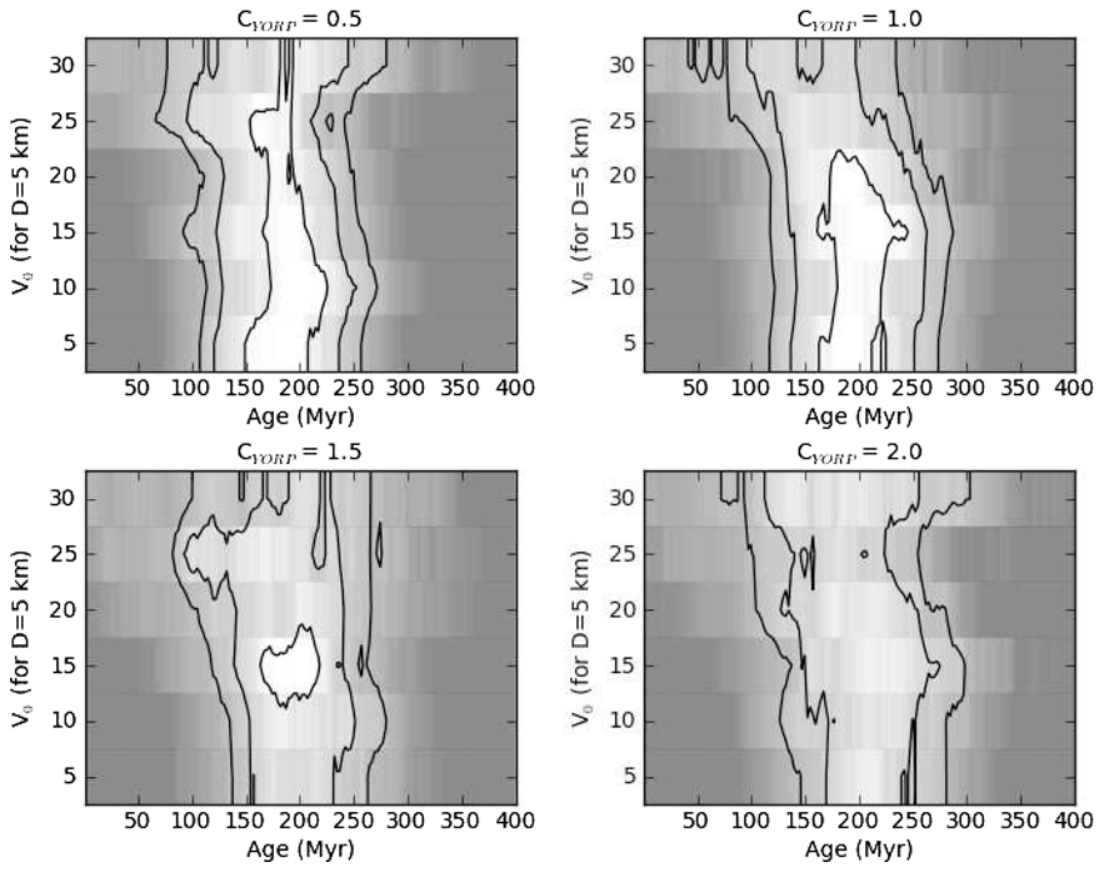

Figure 11. $\chi^{2}$ maps of breakup velocity $V_{0}$ vs. age for the four tested values of $c_{\text {YORP }}$, with white shading representing the best fits and dark shading the worst. Contours show $\chi^{2}$ levels of 6,12 , and 18 , the first of which defines the boundary of the region of acceptable fits.

ones seen by Mainzer et al. (2011c) when comparing taxonomy and albedo, notably for the objects spectrally identified with the $\mathrm{X}$-complex. Without an independent measurement of the density of a significant number of family members, age fits must be performed over the entire viable range of bulk densities. Otherwise, improperly narrow error windows on the best-fit age will be derived. If the family taxonomy can be linked to meteorite analogs, then a smaller window can be used, though the unknown porosity will still induce uncertainty in the density estimate.

We show in Figure 10 simulations of evolution of the Baptistina family, in this case only varying the density assumed for the family members over a range of $1.0<\rho<2.8 \mathrm{~g} \mathrm{~cm}^{-3}$.
All other physical and orbital parameters follow the assumptions used in Section 3.3. The rapid change in best-fitting age for different densities is a result of the weakening of the accelerative kicks in the orbital velocity from the Yarkovsky force (i.e., for an assumed diameter the force will be constant, while the acceleration will be inversely proportional to the mass and thus the density). This is shown by the $\chi^{2}$ minimum best-fitting age $T$ following a general $T \propto \rho^{-1}$ where $\rho \lesssim 2$, above which the best-fitting age increases rapidly.

Bottke et al. (2007) assumed that both the bulk density and surface density of the Baptistina family members were $1.3 \mathrm{~g} \mathrm{~cm}^{-3}$ from their assumption that the spectral taxonomy 

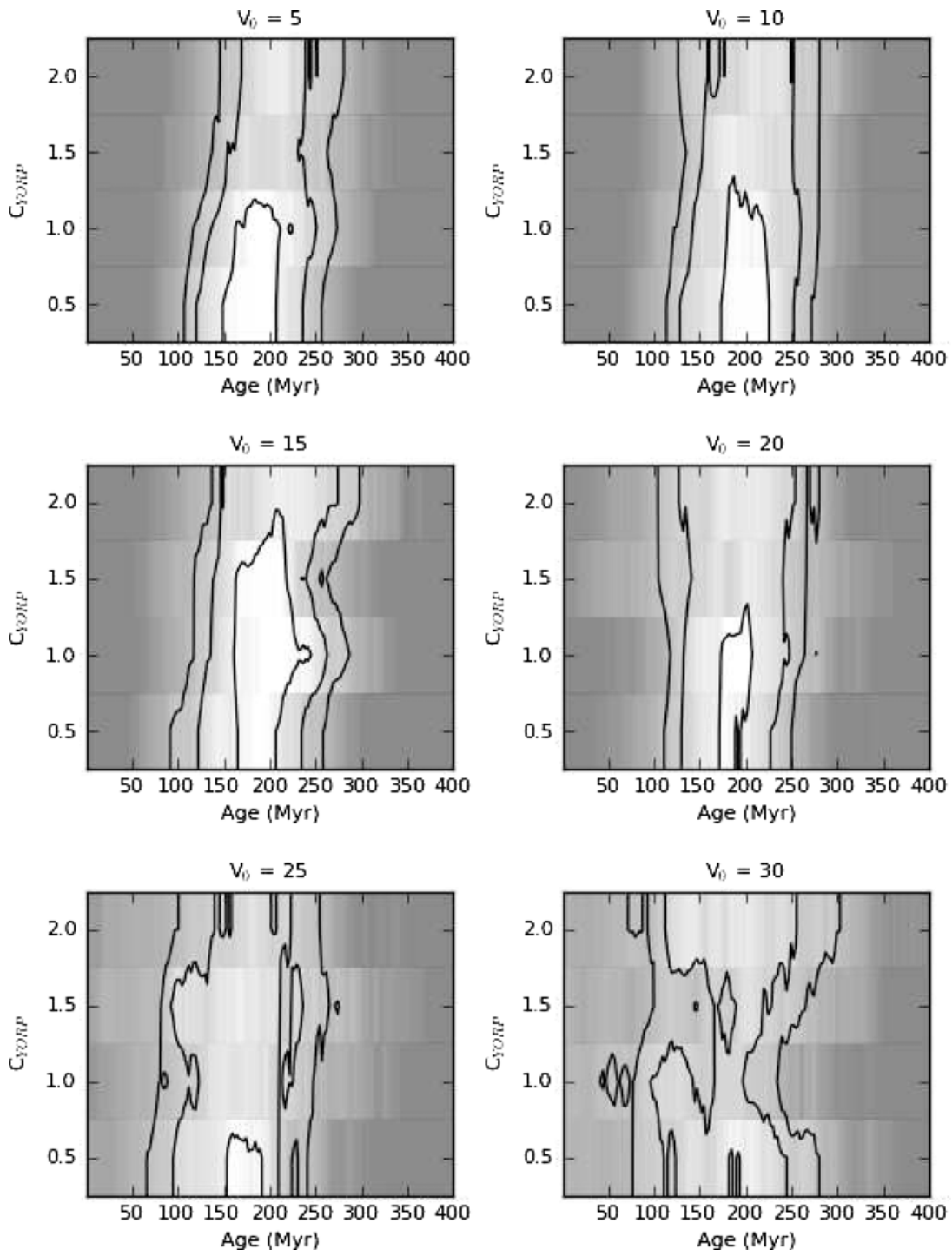

Figure 12. Same as Figure 11, but showing $c_{\text {YORP }}$ vs. age for the six tested values of $V_{0}$.

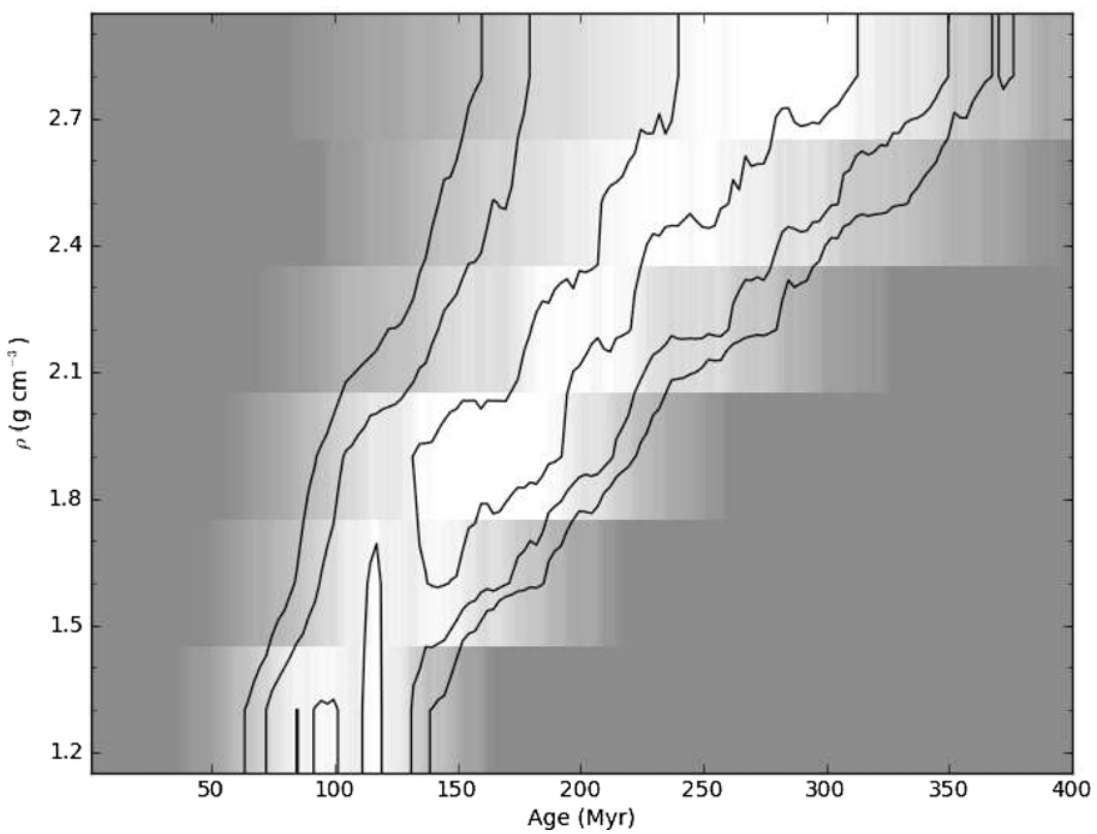

Figure 13. Same as Figure 11, but showing density $\rho$ vs. age assuming the best-fit values of $V_{0}=10 \mathrm{~m} \mathrm{~s}^{-1}$ and $c_{\text {YORP }}=1.0$. 


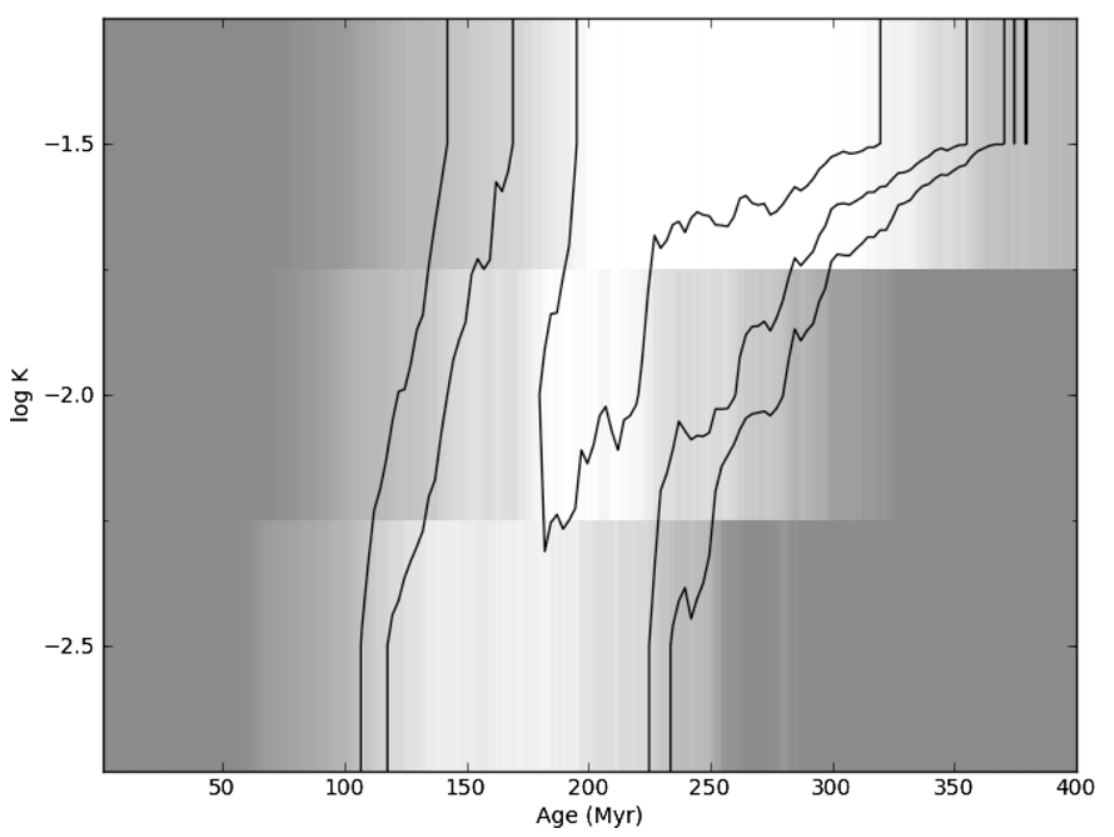

Figure 14. Same as Figure 13 but showing the log of thermal conductivity vs. age.

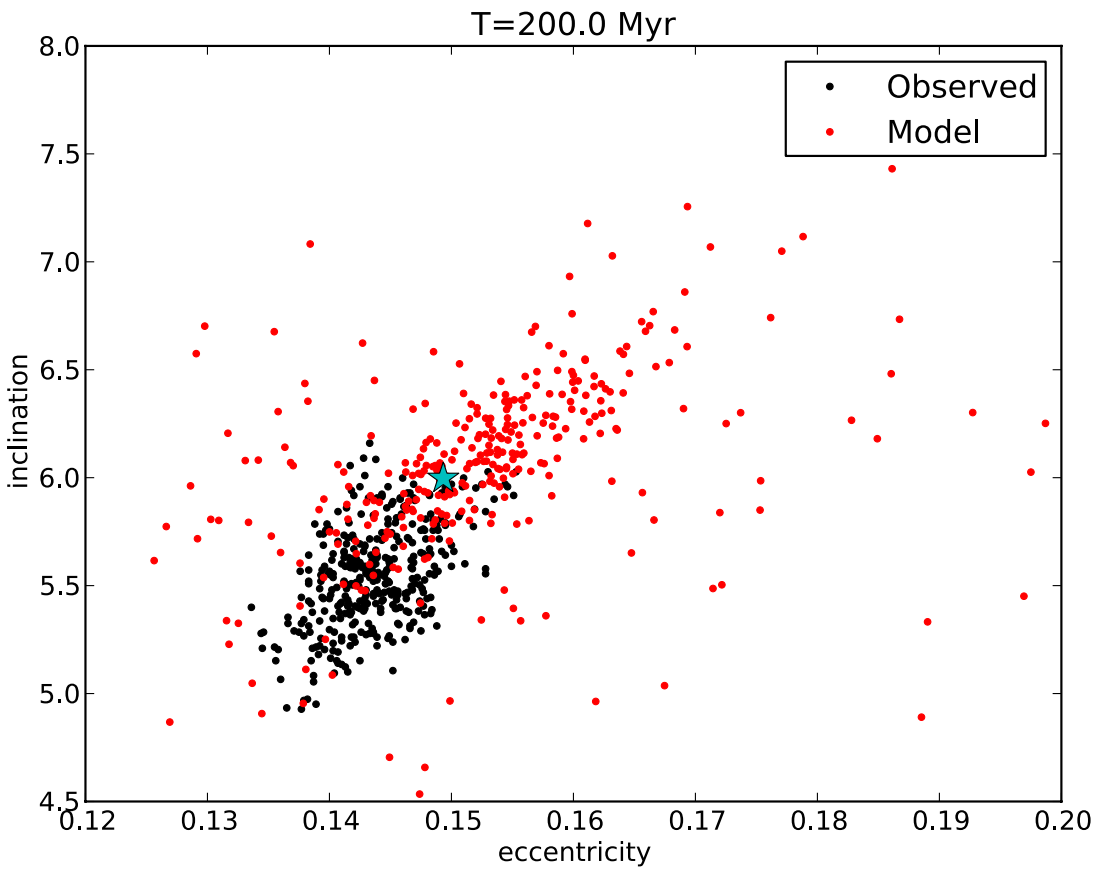

Figure 15. Proper inclination (in degrees) vs. proper eccentricity for the observed Baptistina family members used for our analysis (black) and the simulated model family (red) using the best-fit values of $V_{0}=10 \mathrm{~m} \mathrm{~s}^{-1}, c_{\text {YORP }}=1$, and $T=200 \mathrm{Myr}$ and assumed values of $\rho=2.2 \mathrm{~g} \mathrm{~cm}^{-3}$ and $K=0.01 \mathrm{~W} \mathrm{~m}{ }^{-1} \mathrm{~K}^{-1}$. The cyan star indicates the location of the parent body of the family.

(A color version of this figure is available in the online journal.)

was most similar to a C-type asteroid (however see Reddy et al. 2009,2011 , for further discussion on the taxonomy of Baptistina and its family). For our revised simulations (see Section 4.2), we adopt a bulk and surface density of $2.2 \mathrm{~g} \mathrm{~cm}^{-3}$, assuming S-type taxonomy. However, testing over the full range of probable densities $(\sim 1.6$ to $\sim 2.8)$ will result in a broadening of the best-fit range.

\section{THE AGE OF BAPTISTINA INCORPORATING WISE RESULTS}

Using the methodology developed by Vokrouhlický (1998), we revise the estimated age of the Baptistina family by Bottke et al. (2007) by taking into account the diameter and albedo measurements offered by NEOWISE for $\sim 1 / 3$ of the known family members. One complicating factor in identifying and modeling this family is its partial overlap in orbital element space with the much larger and older Flora family. The albedo distinction between these two families should enable us to use this parameter as a further restriction on family membership, and development, testing, and analysis of this method will be presented in a future paper. For this preliminary analysis, we use a restricted set of family members that includes only the objects that have drifted outward from the parent and thus are not contaminated by Flora, as discussed above in Section 2.5. 

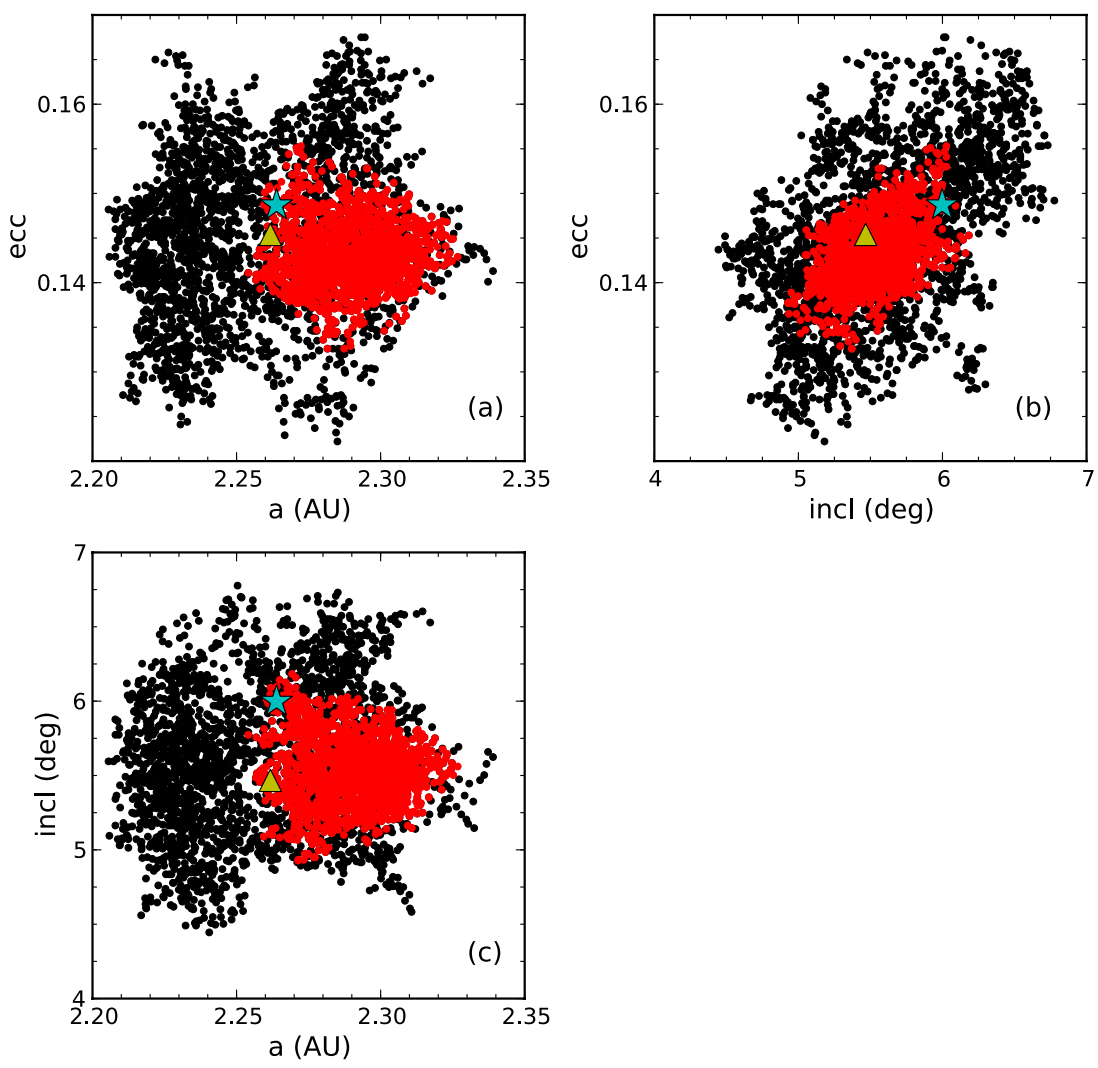

Figure 16. Proper orbital elements for all members of the Baptistina collisional family in black, the restricted family list used for comparison to our simulations in red, (298) Baptistina as the cyan star, and (1696) Nurmela as the yellow triangle.

(A color version of this figure is available in the online journal.)

\subsection{New Observational Data}

The diameters and albedos we use for this work are drawn from the values derived for MBAs published in Masiero et al. (2011). The larger asteroids were more likely to have been seen in multiple bands by WISE which allows for fitting of the beaming parameter. Mainzer et al. (2011b) show that in cases such as this the absolute error on diameter is $\sim 10 \%$ and on albedo is $\sim 20 \%$ of the measured albedo value, however, internal comparisons are better than this limit. We note that this albedo error assumes moderate-to-low light curve amplitudes and well characterized $\mathrm{H}$ and $\mathrm{G}$ values. In addition to observing known objects, NEOWISE also discovered new asteroids, preferentially with lower albedos where ground-based surveys are less sensitive. These previously unknown objects represent a source of error in the diameter and albedo distribution of known families as they would not be included in the known family lists, and will tend to make the true albedo distribution darker than the distribution seen for the previously known asteroids, most of which were discovered by visible light surveys that are biased against detecting low albedo objects. Future work will address the error resulting from this change in albedo distribution.

The primary variation between the observed data and the assumed values in Bottke et al. (2007) is the average value for the albedo of the family measured by $\operatorname{WISE}\left(p_{V}=0.21\right)$ compared with the assumed value used previously $\left(p_{V \text {, assumed }}=0.05\right)$. The main effect of this change is to reduce by more than a factor of two the effective size of a typical Baptistina family member used in simulations. We note that because the albedo distribution of the Baptistina family is fairly wide $( \pm 0.1)$, the change in diameter from assumed to measured values for each individual family member can be much larger or smaller than the factor of two derived from applying the mean albedos. It is therefore critical to use the actual measured diameters for family members where available, instead of assuming a uniform albedo for all objects. This will also remove an additional source of uncertainty that is inherent to the $H$ magnitude measurement.

As discussed in Section 3.4, the density chosen for the test particles can have a very large effect on the best-fit age that is determined for the family. Bottke et al. (2007) use a density of $1.3 \mathrm{~g} \mathrm{~cm}^{-3}$ appropriate for small C-complex bodies (Carry 2012), as Baptistina was thought to be. The revisions in asteroid sizes and albedos from the WISE data, as well as taxonomic classification of a larger set of Baptistina family members as S-complex bodies (Reddy et al. 2011), drives us to assume a larger bulk density for the objects. For our initial simulations, we assume $\rho=2.2 \mathrm{~g} \mathrm{~cm}^{-3}$, however we also test a range of densities using the updated diameters.

\subsection{Revised Age and Error}

Using a set of test particles with the same size and albedo as were measured for the Baptistina family by WISE, we simulate their evolution over $400 \mathrm{Myr}$ using for our initial conditions: present day osculating elements for Baptistina and Venus through Saturn, $\epsilon_{0}=1.0, K_{0}=0.01 \mathrm{~W} \mathrm{~m}^{-1} \mathrm{~K}^{-1}$, $C_{p, 0}=680 \mathrm{~J} \mathrm{~kg}^{-1} \mathrm{~K}^{-1}$, and $\rho_{0}=\rho_{s, 0}=2200 \mathrm{~g} \mathrm{~cm}^{-3}$. We initially test a grid of breakup velocities $\left(V_{0}\right)$ and $c_{\text {YORP }}$ parameters. In Figure 11, we show $\chi^{2}$ maps of $V_{0}$ versus age for

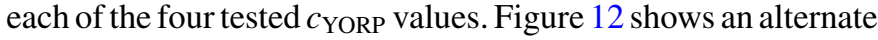
view of the same simulations, with each map showing $c_{\text {YORP }}$ versus age for a given $V_{0}$. For this limited range of assumed 
parameters, the best-fit age is $190 \pm 30 \mathrm{Myr}$, with minimal dependence on $V_{0}$ and $c_{\text {YORP }}$ in the ranges of $5<V_{0}<20 \mathrm{~m} \mathrm{~s}^{-1}$ and $0.5<c_{\text {YORP }}<1.5$, and only a slight preference for lower values in each case. We note that due to the albedo assumed by Bottke et al. (2007) of $p_{V}=0.05$, the inferred diameters are approximately a factor of two larger for the family members and thus it is not unexpected that their best-fit $V_{0} \sim 40$ is similarly larger than the best-fit value we find.

While best-fit age has minimal dependence on $V_{0}$ and $c_{\text {YORP }}$, the assumed value for density and thermal conductivity induce large changes in the final age determination. We show in Figure 13 the $\chi^{2}$ map of density versus age for simulations using $V_{0}=10 \mathrm{~m} \mathrm{~s}^{-1}$ and $c_{\text {YORP }}=1.0$. We note that for $\rho=1.3 \mathrm{~g} \mathrm{~cm}^{-3}$ (the value assumed by Bottke et al. 2007), the best-fit age is $\sim 80 \mathrm{Myr}$ which is consistent with the inverse relation between age and the square root of the assumed albedo as specified by those authors. For a reasonable range of assumed densities of $1.6-2.8 \mathrm{~g} \mathrm{~cm}^{-3}$, we find the best-fitting age can vary from 140 to $320 \mathrm{Myr}$. Without a better constraint on family member density, it will be difficult to more precisely determine the age of the family.

Figure 14 shows a similar test, but now for a varied thermal conductivity in the range of $0.003<K<0.03 \mathrm{~W} \mathrm{~m}^{-1} \mathrm{~K}^{-1}$ (assuming $\rho=2.2 \mathrm{~g} \mathrm{~cm}^{-3}$ ). Larger values of thermal conductivity result in an increase in the best-fit age comparable to the change caused by a larger assumed density. Like density, the fact that thermal conductivity is relatively unconstrained sets a fundamental limit on the accuracy of simulations of family evolution and age.

We note that while our simulations can reproduce the semimajor axis distribution of the family well for a variety of assumed parameters, there are shortcomings to our solution. In particular, we are unable to simulate the observed distribution of the family members in inclination-eccentricity space for any of the range of parameters tested above. We show in Figure 15 the inclination-eccentricity distribution for the observed Baptistina family compared with the family simulated using $V_{0}=$ $10 \mathrm{~m} \mathrm{~s}^{-1}, c_{\text {YORP }}=1, \rho=2.2 \mathrm{~g} \mathrm{~cm}^{-3}, K=0.01 \mathrm{~W} \mathrm{~m}^{-1} \mathrm{~K}^{-1}$, and an age of $T=200 \mathrm{Myr}$. Proper orbital elements are calculated for the simulated particles using a frequency-modified Fourier transform (Š́idlichovský \& Nesvorný 1996) with frequency filters described by Brož (2006). The offset observed between the two populations may indicate that the breakup had an ejection velocity distribution that was highly anisotropic (unlike the assumed isotropic distribution used in our simulations), that the assumed initial orbital parameters for the parent at the time of breakup are incorrect, or that the asteroid identified as the parent body is not the source of the breakup that created the family. As an example, we show in Figure 16 the proper orbital elements of all objects identified as members of the Baptistina family by Nesvorný (2010), the restricted list we use for comparisons to our simulations (as discussed in Section 2.5), (298) Baptistina, and (1696) Nurmela: the largest body at the center of the a-e-i distribution which has a diameter of $D=9.9 \mathrm{~km}$. Future work will investigate these scenarios.

\section{CONCLUSIONS}

Using a symplectic integrator modified to include the effects of gravity, Yarkovsky, and YORP, we have simulated the evolution of a synthetic Baptistina asteroid family from breakup through $\sim 400 \mathrm{Myr}$ of evolution. We compare the distribution at each time step to the observed distribution of the Baptistina family members to determine the age of the family. By varying all assumed parameters, we set constraints on the effect of each parameter on the determined age, and thus the error induced by the uncertainty in its true value.

We find that while most physical parameters do not significantly change our results, both the density and thermal conductivity of the surface can drastically change the best-fit ages resulting in uncertainties greater than $\sim 50 \%$, either younger or older. While having updated values for diameter and albedo reduces the uncertainty in the simulation and the resultant age when compared to models conducted using only absolute magnitude, assumptions for the other physical parameters remain a significant source of uncertainty in the calculation.

Using the WISE-derived albedos and diameters, we find a best-fitting age for the Baptistina family of $190 \pm 30 \mathrm{Myr}$ when we used a single assumed density of $\rho=2.2 \mathrm{~g} \mathrm{~cm}^{-3}$ and an assumed thermal conductivity of $K=0.01 \mathrm{~W} \mathrm{~m}^{-1} \mathrm{~K}^{-1}$. When we allow density and thermal conductivity to vary over nominal ranges ( $\pm 30 \%$, and up or down by a factor of three, respectively), we find that the best-fitting age can range anywhere from 140 to $320 \mathrm{Myr}$.

The differences between our results and the findings of Bottke et al. (2007) are due primarily to the smaller size of the Baptistina family members that we measure compared to their assumed values and the increase in the assumed density. A higher assumed density will weaken the non-gravitational forces compared to gravitational perturbation and slow the overall evolution when strong gravitational interactions do not dominate the process. We also note that the revised albedo and diameter measurements result in a reduction in both the size of the preimpact body and the number of large fragments produced in the impact, decreasing the number available to enter the near-Earth population.

Our simulations all assume that (298) Baptistina is the parent of the Baptistina family and that its orbital elements at the time of breakup were the same as today. If instead a different object is the parent of the family, then the family age may change dramatically from the values found here. A new suite of simulations would be required, using the updated parent, to determine the family age. Future work will explore this possibility for the Baptistina family.

In the end, we are unable to set a firm constraint on the age of the Baptistina family without more information about the family's physical parameters ( $\rho$ and $K$, specifically). However, the uncertainty in this age determination can be greatly reduced with focused investigations of the family members. In particular, thermophysical modeling of a selection of Baptistina family members will allow us to better constrain the physical parameters such as thermal conductivity, while identification and study of any binary asteroids that may be family members will allow us to decrease the uncertainty in the density of those bodies, and by extension the family as a whole. Future work will extend our investigation to the remaining asteroid families observed by WISE, taking into account the caveats and concerns we highlight here.

We thank the referee, Bill Bottke, for his helpful and insightful comments that resulted in a critical reanalysis of the data, greatly improving our results and the manuscript in general. We also thank Bob McMillan for his editing of this manuscript. J.R.M. was supported by an appointment to the NASA Postdoctoral Program at JPL, administered by Oak Ridge Associated Universities through a contract with NASA. Computer simulations for this research were carried out on JPL's Zodiac 
supercomputer, which is administered by the JPL Supercomputing and Visualization Facility. The supercomputer used in this investigation was provided by funding from the JPL Office of the Chief Information Officer. This publication makes use of data products from the Wide-field Infrared Survey Explorer, which is a joint project of the University of California, Los Angeles, and the Jet Propulsion Laboratory/California Institute of Technology, funded by the National Aeronautics and Space Administration. This publication also makes use of data products from NEOWISE, which is a project of the Jet Propulsion Laboratory/California Institute of Technology, funded by the Planetary Science Division of the National Aeronautics and Space Administration. This research has made use of the NASA/IPAC Infrared Science Archive, which is operated by the Jet Propulsion Laboratory, California Institute of Technology, under contract with the National Aeronautics and Space Administration.

\section{REFERENCES}

Alvarez, L. W., Alvarez, W., Asaro, F., \& Michel, H. V. 1980, Science, 208, 1095

Bottke, W. F., Vokrouhlický, D., \& Nesvorný, D. 2007, Nature, 449, 48

Bottke, W. F., Vokrouhlický, D., Rubincam, D. P., \& Nesvorný, D. 2006, Annu. Rev. Earth Planet. Sci., 34, 157

Brož, M. 2006, PhD thesis, http://sirrah.troja.mff.cuni.cz/ mira/mp/phdth/

Capek, D., \& Vokrouhlický, D. 2004, Icarus, 172, 526

Carry, B. 2012, P\&SS, in press (arXiv:1203.4336v1)

Ćuk, M., Gladman, B. J., \& Stewart, S. T 2010, Icarus, 207, 590

Delbo, M., \& Tanga, P. 2009, Planet. Space Sci., 57, 259

dell'Oro, A., Paolicchi, P., Cellino, A., \& Zappalà, V. 2002, Icarus, 156, 191

Farinella, P., Vokrouhlický, D., \& Hartmann, W. K. 1998, Icarus, 132, 378

Farley, K. A., Vokrouhlický, D., Bottke, W. F., \& Nesvorný, D. 2006, Nature, 439,295

Hirayama, K. 1918, AJ, 31, 185
Le Feuvre, M., \& Wieczorek, M. A. 2011, Icarus, 214, 1

Levison, H. F., Dones, L., Chapman, C. R., et al. 2001, Icarus, 151, 286

Levison, H. F., \& Duncan, M. J. 1994, Icarus, 108, 18

Mainzer, A. K., Bauer, J. M., Grav, T., et al. 2011a, ApJ, 731, 53

Mainzer, A. K., Grav, T., Masiero, J., et al. 2011b, ApJ, 736, 100

Mainzer, A. K., Grav, T., Masiero, J., et al. 2011c, ApJ, 741, 90

Masiero, J., Jedicke, R., Durech, J., et al. 2009, Icarus, 204, 145

Masiero, J., Mainzer, A. K., Grav, T., et al. 2011, ApJ, 741, 68

Morbidelli, A., Brasser, R., Gomes, R., Levison, H. F., \& Tsiganis, K. 2010a, AJ, 140,1391

Morbidelli, A., Brasser, R., Nesvorny, D., Vokrouhlicky, D., \& Bottke, W. F. 2010b, BAAS, 42, 408

Nesvorný, D. 2010, EAR-A-VARGBDET-5-NESVORNYFAM-V1.0, NASA Planetary Data System

Nesvorný, D., \& Bottke, W. F. 2004, Icarus, 170, 324

Nesvorný, D., Bottke, W. F., Vokrouhlický, D., Morbidelli, A., \& Jedicke, R. 2006, in Proc. IAU Symp. 229, Asteroids, Comets, Meteors, ed. L. Daniela, M. Sylvio Ferraz, \& F. J. Angel (Cambridge: Cambridge Univ. Press), 289

Nesvorný, D., Jedicke, R., Whiteley, R. J., \& Ivezić, Ž 2005, Icarus, 173, 132

Nesvorný, D., Morbidelli, A., Vokrouhlický, D., Bottke, W. F., \& Brož, M. 2002, Icarus, 157,155

O’Brien, D. P., \& Greenberg, R. 2005, Icarus, 178, 179

Reddy, V., Carvano, J. M., Lazzaro, D., et al. 2011, Icarus, 216, 184

Reddy, V., Emery, J. P., Gaffey, M. J., et al. 2009, Meteorit. \& Planet. Sci, 44, 1917

Rubincam, D. P. 2000, Icarus, 148, 2

S̆idlichovský, M., \& Nesvorný, D. 1996, Celest. Mech. Dyn. Astron., 65, 137

Tedesco, E. F., Noah, P. V., Noah, M., \& Price, S. D. 2002, AJ, 123, 1056

Tsiganis, K., Gomes, R., Morbidelli, A., \& Levison, H. F. 2005, Nature, 435,459

Vokrouhlický, D. 1998, A\&A, 335, 1093

Vokrouhlický, D. 1999, A\&A, 344, 362

Vokrouhlický, D., Brož, M., Bottke, W. F., Nesvorný, D., \& Morbidelli, A. 2006, Icarus, 182, 118

Vokrouhlický, D., \& Čapek, D. 2002, Icarus, 159, 449

Vokrouhlický, D., \& Farinella, P. 1999, AJ, 118, 3049

Wright, E. L., Eisenhardt, P., Mainzer, A. K., et al. 2010, AJ, 140, 1868

Zappalà, V., Cellino, A., Farinella, P., \& Knezevic, Z. 1990, AJ, 100, 2030

Zappalà, V., Cellino, A., Farinella, P., \& Milani, A. 1994, AJ, 107, 772 